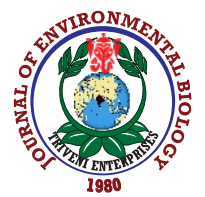

\title{
Molecular characterization of endophytic fungal communities associated with Vitis vinifera L. at Taif region of Saudi Arabia
}

\author{
S.M. Sayed ${ }^{1,2, *}$, A.M. El-Shehawi ${ }^{3,4}$, S.A. Elarnaouty ${ }^{2}$, S.A. Al-Otaibi ${ }^{3}$, S.A. El-Shazly ${ }^{3,5}$, S.S. Alotaibi ${ }^{3}$, R. Ibrahim ${ }^{6,7}$, M.M. Ahmed $^{8}$ and M.M. Elseehy ${ }^{4}$ \\ 'Department of Science and Technology, University College of Ranyah, Taif University, Taif-21974, SaudiArabia \\ ${ }^{2}$ Department of Economic Entomology, Faculty of Agriculture, Cairo University, Giza-12613, Egypt \\ ${ }^{3}$ Department of Biotechnology, Faculty of Science, Taif University, Taif-21974, Saudi Arabia \\ ${ }^{4}$ Department of Genetics, Faculty of Agriculture, Alexandria University, Alexandria-21526, Egypt \\ ${ }^{5}$ Department of Biochemistry, Faculty of Veterinary Medicine, Kafrelsheikh University, Kafr El-Sheikh 33511, Egypt \\ ${ }^{6}$ Department of Biology, Faculty of Science, Taibah University, Medina 344, Saudi Arabia \\ ${ }^{7}$ Department of Economic Entomology, Faculty of Agriculture, Kafrelsheikh University, Kafr El-Sheikh 33516, Egypt \\ ${ }^{8}$ Department of Biochemistry, Faculty of Veterinary Medicine, University of Sadat City, 32958, Egypt \\ *Corresponding Author Email : samy_mahmoud@hotmail.com
}

\section{Abstract}

Aim: The current study aimed to characterize the composition of endophytic fungal communities related to Taify grapevine leaves.

Methodology: Taify grapevine leaves were collected from three different locations at Taif region. Total DNA was extracted and the endophytic fungal communities were investigated using next generation sequencing in a metagenomics approach.

Results: The obtained data detected 26 fungal operational taxonomic units (OTUs) in all locations with variation in most taxa among three locations. The phylum Ascomycota dominated relative sequence abundance $(90.83 \%)$, followed by Basidiomycota at $1.82 \%$. Other unidentified fungi at phylum level dominated $7.35 \%$ abundance level. At genus level, Alternaria and Cladosporium genera were present at all locations, while Filobasidium and Didymella were detected in two locations. Most genera, Penicillium, Hanseniaspora, Fusarium, Chrysocorona and Quambalaria were detected in one location only.

Interpretation: The present study confirmed the diversity of endophytic fungi in Taify grapevine, including pathogenic and nonpathogenic species. The detected species have potential to be used as biocontrol species against economically important pathogens such as Alternaria and Hanseniaspora. This will contribute to our understanding of grapevine-endophytic fungi interaction and their utilization to enhance grapevine production and quality.

Key words: Endophytes, Fungi, Grapevine, Metagenomic, Next generation sequencing

How to cite : Sayed, S.M., A.M. El-Shehawi, S.A. Elarnaouty, S.A. Al-Otaibi, S.A. El-Shazly, S.S. Alotaibi, R. Ibrahim, M.M. Ahmed and M.M. Elseehy: Molecular characterization of endophytic fungal communities associated with Vitis vinifera L. at Taif region of Saudi Arabia. J. Environ. Biol., 42, 177-185 (2021).

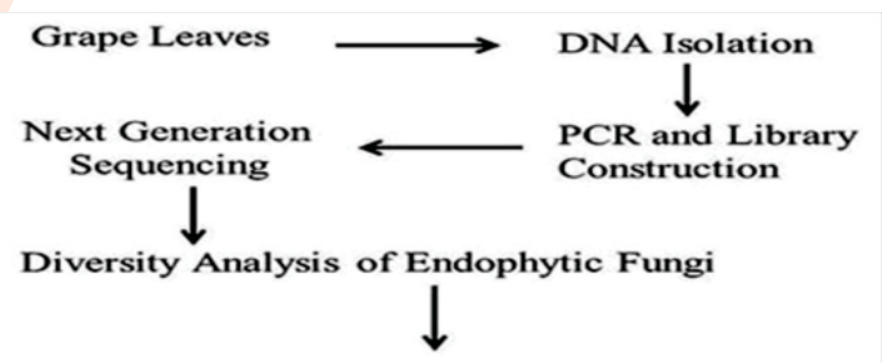

Detected endophytic fungi will contribute in the biocontrol of grape pests and enhancement of grape production and quality 


\section{Introduction}

Grapevine (Vitis vinifera L.) is a woody perennial plant and is widely cultivated in different regions of the world. It is an economically important fruit crop where its fruit is used as table grapes, or production of raisins, juice and wine. At least 70 intracellular infectious pathogens infect grapevines, exclusive of fungi, that the most registered for any other single crop (Martelli, 2014). In Saudi Arabia, it is the second most important economical fruit which is consumed as table grapes, grape juice, or raisins. A variety that is cultivated in Taif region (high altitude region) is called Taif's grape where the environment is suitable for its cultivation (Fahmi et al., 2012). Endophytic microorganisms including fungi are present within the tissues of host plant and do not cause apparent disease symptoms. Several species enhance the plant's growth by producing growth-stimulating substances such as phytohormones or by increasing the uptake of minerals such as nitrogen and phosphorus (Hernández-Hernández et al., 2018). Some endophytes have other potential benefits for their host plants such as tolerance to abiotic stress, defense against bacterial and fungal pathogens, parasites or herbivores, by producing functional metabolites that have various toxic properties against these organisms (Gonzalez and Tello, 2011). Accordingly, endophytes have been used in the biological control programs of insect pests, plant diseases and parasitic nematodes (Hallmann and Sikora, 1996).

Investigation of endophytic communities in grapevine varieties is essential for optimization of future pest management and high quality production (Varanda et al., 2016). There are different methods to study the microorganism's community profiling such as terminal restriction fragment length polymorphism (T-RFLP) and denaturing gradient gel electrophoresis (DGGE). The culture-independent methods in combination with culturing techniques do not give direct taxonomic identities of the species contributing to the signals. Metagenomics is a technique to examine the entire microbial communities that are present in an environment through investigation of nucleotide sequence content. In this way, NGS is used for estimating relative occurrence of microorganisms in an environment where it produces high amount of sequence data in a single run, followed by direct sequencing without cloning.

Moreover, the utilization of universal primers permits to sequence the mixed genetic material of an environmental sample in an impartial way (Mardis, 2008). Metabarcoding studies target the partial internal transcribed spacer 1 (ITS1) or ITS2 sub-region resulting in lower taxonomic resolution due to shorter reads (do not permit full-length ITS), which is considered as a disadvantage of NGS (Lindahl et al., 2013). During the last two decades, the internal transcribed spacer (ITS1, 5.8 rDNA and ITS2) of nuclear DNA has been used as a standard marker for fungal DNA barcoding and analyzing the fungal diversity in different environmental samples (Srivastava et al., 2017). In this way, researchers have used various universal primers for amplifying this region, especially ITS1 and ITS4 in order to characterize and monitor diversity of fungi including endophytes (Varanda et al., 2016; Brum et al., 2012; Setati et al., 2012; Hamzah et al., 2018). Endophytic diversity is heavily affected by the environment, plant species, type of soil, and many other factors. Endophytic fungal community of Taify grape has not been investigated at the level of metagenome diversity and richness. Therefore, the aim of the current study was to characterize the composition of endophytic fungal communities associated with Taify grapevine leaves in Taif region of Saudi Arabia using next generation sequencing via metagenomic approach.

\section{Materials and Methods}

Study site and collection of plant material: Three different namely located in Taif, Saudi Arabia was selected as study site. $L 1, L 2$ and L3. These locations are cultivated with 3-year-old grapevine plants (Vitis vinifera cv. 'Taify'). In September 2019, from each location, three distant plants were randomly selected in order to collect three healthy leaves from approximately height of 1.0, 1.5 and $2.0 \mathrm{~m}$ above the ground and transferred to the laboratory. All leaf samples were surface sterilized in $70 \%$ ethanol for $45 \mathrm{sec}, 1 \mathrm{~min}$ in sodium hypochlorite, $45 \mathrm{sec}$ in $70 \%$ ethanol, and washed four times in autoclaved sterilized water. Samples were lyophilized at- $58^{\circ} \mathrm{C}$ for $48 \mathrm{~h}$, and then three leaves from each plant were ground together (as one sample) into fine powder in TissueLyzer II (Qiagen, Germany). Ground samples were kept at $-2^{\circ} \mathrm{C}$ until further use.

Isolation of DNA: DNA was isolated with lyophilized powder from all the samples of different locations $(L 1, L 2, L 3)$ by the CTAB method. One $\mathrm{ml}$ of CTAB extraction buffer was used to resuspend $5 \mathrm{mg}$ of sample powder in $1.5 \mathrm{ml}$ tube and mixed thoroughly at $65^{\circ} \mathrm{C}$. The mixture was incubated for $2 \mathrm{hr}$ at $65^{\circ} \mathrm{C}$ in a shaking water bath. Chloroform: Isoamylalchohol, was used to extract samples twice. DNA was precipitated from the supernatant using 1 volume of isopropanol, then centrifugated for $10 \mathrm{~min}$ at $12000 \mathrm{rpm}$ at $4^{\circ} \mathrm{C}$. DNA pellet was washed with 0.5 $\mathrm{ml}$ of $70 \%$ ethanol, dried, and reconstituted in sterilized water. RNA was removed from DNA samples by adding $1 \mu$ Ribonuclease $A$ and incubated at $37^{\circ} \mathrm{C}$ for $20 \mathrm{~min}$.

DNA quantification: DNA was estimated using Picogreen method (Invitrogen, cat.\#P7589) and Victor 3 fluorometry. This florescence-based method is preferred over UV absorbance method because it estimates double stranded DNA more accurately in the presence of other contaminants, especially RNA which gives overestimation of DNA concentration. DNA quality and integrity was evaluated by gel electrophoresis.

PCR amplification and CDNA library construction: Fungal metagenome was obtained using PCR targeting the ITS (ITS15.8S rDNA-ITS2) region with the universal eukaryotic primers of ITS1:5'-TCCGTAGGTGAACCTGCGG-3' and ITS4: 5'TCCTCCGCTTATTGATATGC-3' (White et al., 1990). For amblicon library construction, PCR products were indexed with end adaptors using Nextera XT Index kit (Illumina, USA). 
Products were cleaned with AMPure XP beads and used for PCR amplification. The amplified products were tested for correct expected size and cleaned with AMPure XP beads.

Library quantification and sequencing: Library quantification and sequencing were conducted at Macrogen Company (South Korea). Original libraries were diluted and equal aliquots of individual libraries were mixed giving the pooled library. The quality of each library was checked on Bioanalyzer DNA 1000 chip using a 1:50 diluted portion of the library. MiSeq paired end platform (Illumina, USA) was employed to sequence the pooled libraries for each location using the setting; $300 \mathrm{bp}, 5 \%$ Phi X, 100,000 paired end reads per library, using the $d$ MiSeq Reporter software (MSR). This read number is sufficient for saturation of fungal species in different samples. The MiSeq program uses Green genes database to distribute the obtained fungi based on the ITS sequence into various levels of taxonomy starting from kingdom to species. The quality of obtained sequences was checked using CD-HIT-OTU/rDNA Tools software. This included filtration of bad and short reads, removing adaptors and chimeras, and finally clustering the trimmed sequences into Operational Taxonomic Units (OTUs). The trimmed paired end reads were assembled into FastQ file using FLASH.

Diversity analysis: Diversity estimation among grape endophytic fungi at three studied location was achieved by QIIME. Various programs of this package directly use the obtained raw data from llumina platforms. OTUs were assigned into taxonomic units using BLAST integrated in the QIIME package (Caporaso et al., 2010). Rarefication curves were employed to unify the sequencing depth and read counts in all samples to uncover all possible endophytic fungi (Kuczynski et al., 2011). Alpha diversity was estimated using alph_diversity.py. This included estimation of the observed OTUs, Chao1 expected
OTUs, Shannon Index and Simpson Index (Kim et al., 2017). PCoA 2D distribution was ploted by Makes_2d_plots.py to estimate the principle coordinate analysis $(\overline{P C O A})$ of three locations based on their sequence analysis. The software UPGMA_cluster.py was utilized to establish the UPGMA phylogenetic relationship. Finally, taxonomy composition was investigated by UCLSUT, where the rarefaction curve graph was established using alpha_rarefaction.py program.

\section{Results and Discussion}

The quality of DNA library of $L 1, L 2, L 3$ revealed that the average size was 583,580 , and 586 bp consecutively. As shown in Fig. (1), the lower limit and the upper limit of DNA size in L1, L2, L3 locations were $25 \mathrm{bp}$ and $1500 \mathrm{bp}$, respectively, with all of the three libraries size peaking at their respective size. This confims the homogenity and high quality of targeted amplicon libraries across samples. Quality of data is a detrimental factor of final data and data interpretation. The total number of read bases after quality filtering were $64.9,64.5,52.6$ million in L1, L2, L3 groups consecutively. Total read number was 161156 for $L 1,160292$ for L2, 130625 for $L 3$. The GC\% content ranged from 65.3 (L3) to 65.6 (L1), whereas AT content \% ranged from $34.4 \%$ (L1) to $34.7 \%$ (L3) indicating high GC content in Taif grapes.

Phred quality estimated the quality of each read base during sequencing and indicated as $Q$. It was measured by $Q 20$ and Q30 which indicated the error of reading a base 1:100 or 1:1000, respectively. More than $98 \%$ of the read bases were above phred quality 20 (Q20) and more than 93\% of read bases were above phred quality $30(\mathrm{Q} 30)$. These quality measures conclude that the conducted read count was enough for three groups. PCoA provides 2D distribution of groups based on the collective sequence difference among them estimated as principal

Table 1: Taxonomy abundance and ratio of detected endophytic fungi from grape leaves collected from $L 1, L 2$, and $L 3$ locations

\begin{tabular}{|c|c|c|c|c|c|c|c|c|}
\hline \multirow{2}{*}{ Phylum } & \multirow{2}{*}{ Class } & \multirow{2}{*}{ Order } & \multirow{2}{*}{ Family } & \multirow{2}{*}{ Genus } & \multirow{2}{*}{ Species } & \multicolumn{3}{|c|}{ Ratio (\%) } \\
\hline & & & & & & L1 & L2 & L3 \\
\hline & Unidentified & Unidentified & Unidentified & Unidentified & fungal endophyte & 2.13 & 0.98 & 1.01 \\
\hline \multirow[t]{6}{*}{ Unidentified } & Unidentified & Unidentified & Unidentified & Unidentified & uncultured fungus & 48.94 & 8.22 & 1.73 \\
\hline & Dothideomycetes & Capnodiales & Cladosporiaceae & Cladosporium & Cladosporium sp. & 13.83 & 24.07 & 0.91 \\
\hline & & Pleosporales & Didymellaceae & Didymella & D. microchlamydospora & 3.19 & 13.89 & 0.00 \\
\hline & & & Pleosporaceae & Alternaria & A. gaisen & 4.25 & 10.57 & 0.18 \\
\hline & Eurotiomycetes & Eurotiales & Aspergillaceae & Penicillium & P. fagi & 0.00 & 0.00 & 0.27 \\
\hline & & & & Penicillium & P. olsonii & 0.00 & 0.00 & 0.82 \\
\hline \multirow[t]{6}{*}{ Ascomycota } & Leotiomycetes & Erysiphales & Erysiphaceae & Unidentified & Unidentified & 0.00 & 41.88 & 0.00 \\
\hline & Saccharomycetes & Saccharomycetales & Saccharomycodaceae & Unidentified & $\begin{array}{l}\text { uncultured } \\
\text { Hanseniaspora }\end{array}$ & 0.00 & 0.00 & 34.00 \\
\hline & & & & Hanseniaspora & H. vineae & 0.00 & 0.00 & 59.71 \\
\hline & Sordariomycetes & Hypocreales & Nectriaceae & Fusarium & Fusarium sp. & 7.45 & 0.00 & 0.00 \\
\hline & & Sordariales & Chaetomiaceae & Chrysocorona & C. lucknowensis & 0.00 & 0.00 & 0.46 \\
\hline & Exobasidiomycetes & Microstromatales & Quambalariaceae & Quambalaria & Q. cyanescens & 0.00 & 0.00 & 0.27 \\
\hline \multirow[t]{2}{*}{ Basidiomycota } & Tremellomycetes & Filobasidiales & Filobasidiaceae & Filobasidium & F. magnum & 20.21 & 0.00 & 0.27 \\
\hline & Unidentified & Unidentified & Unidentified & Unidentified & Unidentified & 0.00 & 0.39 & 0.36 \\
\hline
\end{tabular}



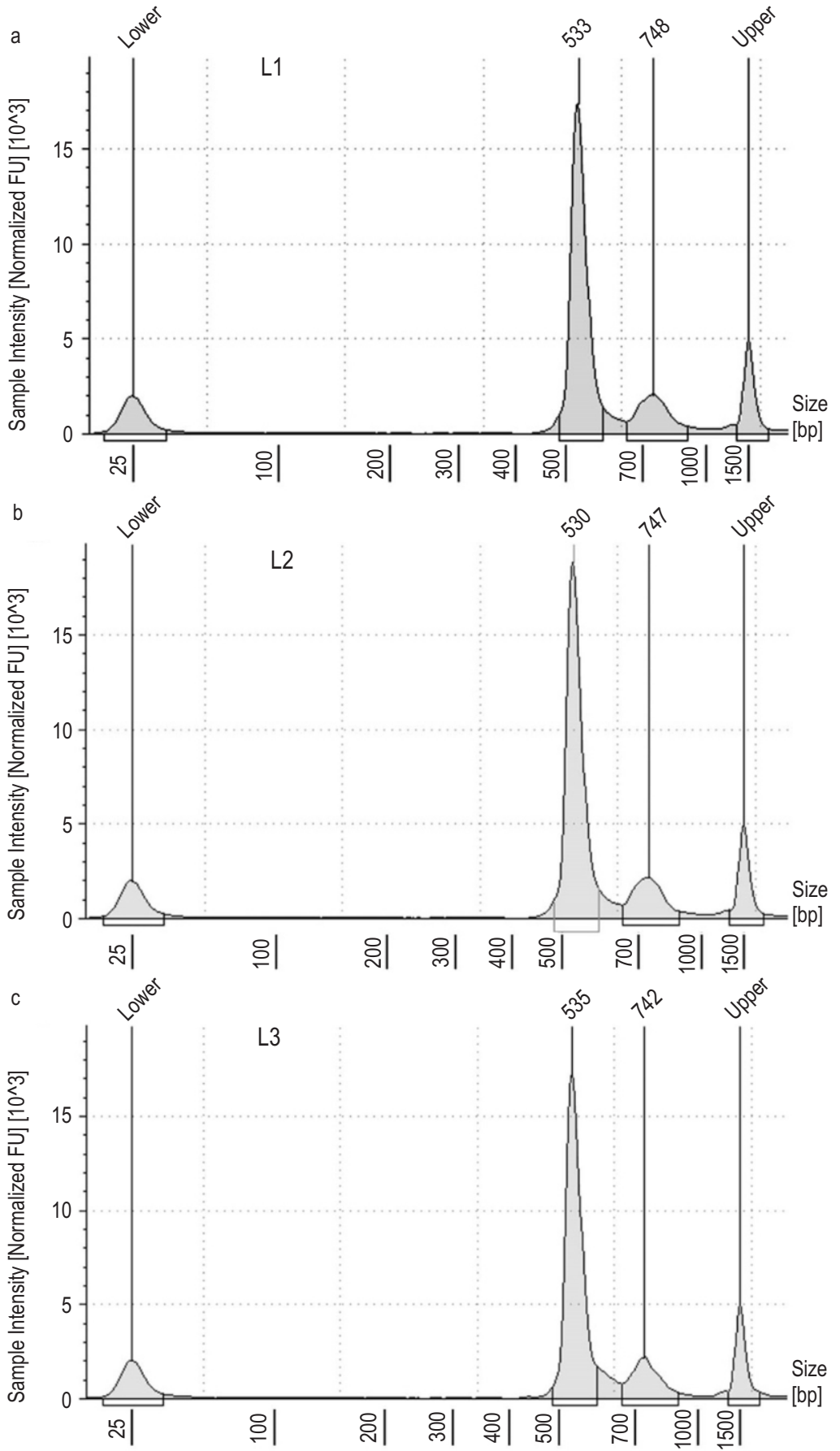

Fig. 1: Quality and size of PCR amplified fungal DNA library of endophytic fungi detected in grape leaves collected from three different geographical locations (L1, L2, L3).

components (PCs). Variations among the ITS amplicon were distributed among three main components namely PC1, PC2, PC3 with $79.67 \%, 20.33 \%$, and $0 \%$ variations, respectively. (Fig. $2 a, b, c)$. The relationship between PC1 and PC2, the main components of variation, distributed three locations apart in both horizontal and vertical dimension (Fig. 2a). This 2D distribution also indicated that based on PC1, L1 was closer to L2 and both were different from L3. On the other hand, based on PC2, three locations were almost vertically equidistant from each other indicating that $\mathrm{PC} 1$ contributes more to the sequence variations 

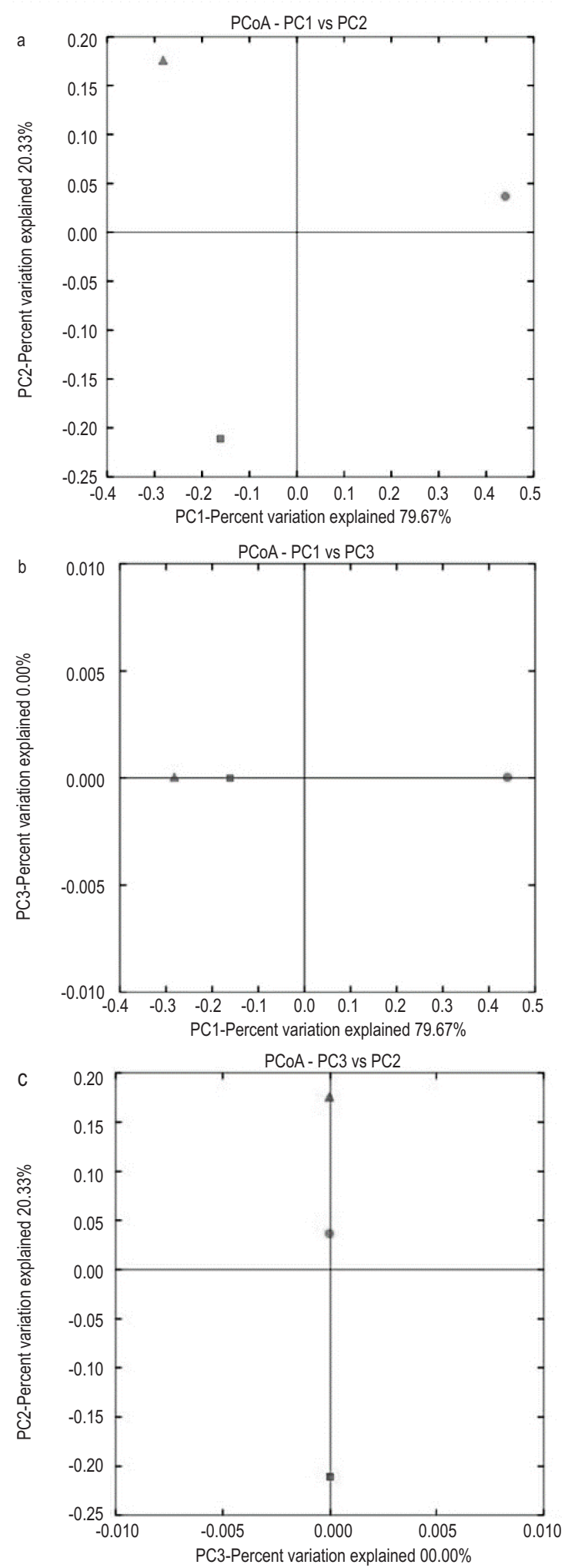

Fig. 2: Principle coordinate analysis among the endophytic fungi detected in grape leaves collected from three different geographical locations (L1, L2, L3). (a) PC1 vs PC2, (b) PC1 vs Pc3 and (c) PC3 vs PC2. L1: red triangle, L2: blue sphere, L3: orange diamond. among three locations (Fig. 2a). Plotting PC1 against PC3 indicated that locations were separated horizontally in the same way in Fig. 2a, whereas they were not separated vertically as PC3 caused $0 \%$ difference among three locations (Fig. 2b). Plotting PC3 against PC2 showed only vertical distribution of locations by PC2 (20.33\% of variations), where $0 \%$ variations due to PC3 did not separate locations horizontally (Fig. 2c). Similar results of PCA analysis were obtained where PCA separated the bacteria of ascites from the bacteria of blood (Wu et al., 2018). Phylogenetic relationship among three locations was established based on the sequence difference in the ITS amplicon for endophytic fungi. L1 and L2 were more related than L3 to any of them. Over 0.35 genetic distance, $\mathrm{L} 1$ and $\mathrm{L} 2$ diverged at 0.2 and the distance from their divergence to L3 was 0.15 indicating that the set of endophytic fungi identified in L3 was significantly different from the detected set in L1 and L2 (Fig. 3). Alpha diversity estimators including Chao OTU and two diversity indices (Shannon, Simpson) are commonly used to estimate the OTUs and microbial community diversity, respectively.

OTU measures the observed OTUs, while Chao estimates the expected OTUs. There was no difference between the observed OTUs and the Chao expected OTUs giving identical detected OTUs at 7, 7, and 12 in the L1, L2, and L3 locations, respectively, with a total 26 OTUs in three locations from 452,073 total read counts (in average, about 17,387 read counts per OTU). On the other hand, other investigations especially in grapevines recorded higher numbers of fungal OTUs (Gonzalez and Tello, 2001; Varanda et al., 2016). This maybe related to high altitude areas where Taif region is approximately $1,800 \mathrm{~m}$ above sea-level and the climate is far cooler with low humidity. In this sense, both fungal composition and diversity were mainly influenced by the vineyard region where the climate areas were colder and drier gave lower fungal species richness in the highest altitude regions (Kioroglou et al., 2019). Also, changes in microbial diversity and composition due to altitude and geographical orientation were recorded (Gao et al., 2019). Shannon Index was 2.25, 2.15, and 1.42 in L1, L2, and L3 locations, respectively. These values indicated that, although L1 and L2 had lower number of endophytic fungi they were more diverse than the endophytic fungi detected in L3. Similarly, the inverse Simpson Index showed more diversity in locations L1 and L2 as compared to L3. Association between the values of Shannon and Inverse Simpson Indices substantially validated the obtained data.

Abundance of different taxonomic levels revealed some interesting features among the three locations. At the phylum level, phylum Ascomycota was highly represented with high relative sequence abundance in $\mathrm{L} 2(90.41 \%)$ and $\mathrm{L} 3(96.35 \%)$, whereas it was underrepresented in L1 $(28.72 \%)$. On the other hand, Basidiomycota was highly represented in L1 than L2 and $L 3$. One general phenomenon was that $L 1$ had higher unidentified ratio at all taxonomic levels as compared to $\mathrm{L} 2$ and $\mathrm{L} 3$ (Table1, Fig. 4). Regardless of location, the phylum Ascomycota dominated relative sequence abundance $(90.83 \%)$, followed by Basidiomycota at $1.82 \%$. Other unidentified fungi at phylum level dominated at $7.35 \%$ abundance level. In general, the phylum 


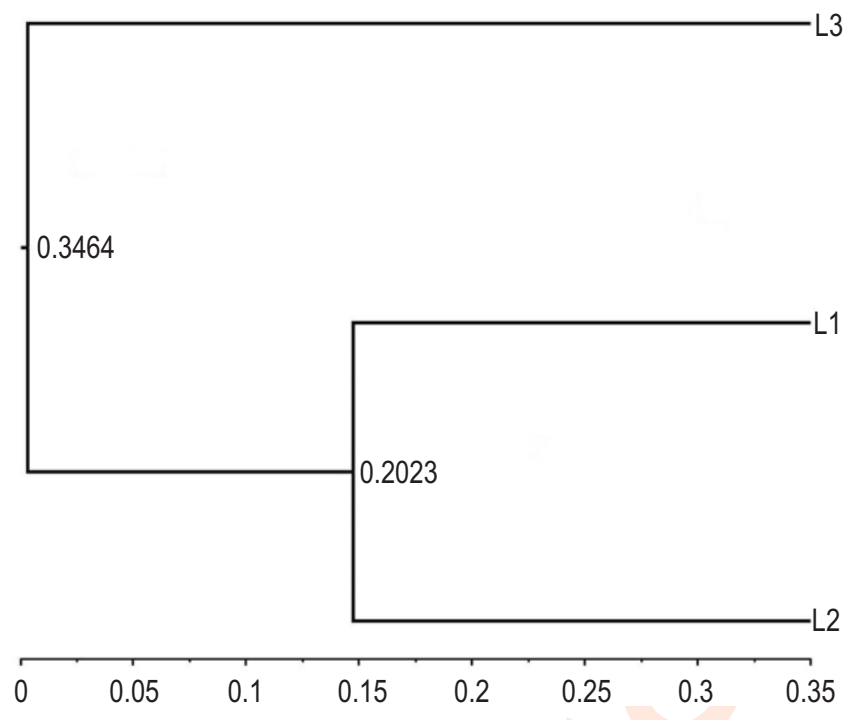

Fig. 3: Phylogenetic tree and genetic distances among the endophytic fungi detected in grape leaves collected from three different geographical locations (L1, L2, L3). Scale below the chart is the genetic distance.

Ascomycota is apparently the most common representative of endophytic fungi community in grapevine (Gonzalez and Tello, 2011; Varanda et al., 2016; Brum et al., 2012) and other woody plants (Hamzah et al., 2018). Basidiomycota phylum in this study represented $1.82 \%$ of relative sequence abundance. In this way, it represented 3.8\% in mangrove (Hamzah et al., 2018), and 3.9\% in strawberry (Abdelfattah et al., 2016). At the class level, Tremellomycetes and Dothideomycetes were the major classes detected in L1, Dothideomycetes and Leotiomycetes were the major classes detected in L2, and Saccharomycetes was the predominant class in L3. Order Filobasidiales and Capnodiales were well represented in L1, Erysiphales, Capnodiales, and Pleosporales orders were highly represented in L2, whereas Saccharomycetales was the revealing order in L3 (Table1, Fig. 4).

At the family level, Filobasidiaceae and Cladosporiaceae were the predominant families in L1, Erysiphaceae and Cladosporiaceae were the prevailing families in $\mathrm{L} 2$, while Saccharomycodaceae was the prevailing family in L3 (Table. 1, Fig. 4). Within Ascomycota, although Saccharomycetes was detected in one location only, it had the highest relative abundance in all data set $(60.3 \%)$, followed by Dothideomycetes $(16.4 \%)$, Sordariomycetes $(12.6 \%)$, Leotiomycetes $(0.7 \%)$ and Eurotiomycetes $(0.7 \%)$ in all data set. In contrast, Dothideomycetes was the dominant class in other studies conducted on grapes (Varanda et al., 2016). Class Saccharomycetes mainly represented by the genus Hanseniaspora was detected in one location, while class Dothideomycetes was represented by the genera Cladosporium, Didymella and Alternaria. At the genus level, Hanseniaspora was the main genus although it was present in only one location. Reports on genus Hanseniaspora as fungal endophytes of $V$. vinifera leaves are meagre, yet it was previously detected as endophyte in different stage of grape berries (Hall and Wilcox, 2018), in both forests and vineyards phyllosphere (Castañeda et al., 2018). Ten species of the genus Hanseniaspora have been recovered in mature grapes and play an important role at initial stage of fermentation, producing aroma compounds and enzymes that expand the diversity of wine color and flavor (Martin et al., 2018). On the other hand, this genus was detected as endophytic in leaves of other plant species. For examples, three species of Hanseniaspora $(H$. uvarum, $H$. thailandica and $H$. opuntiae) were detected as endophyte in leaves of Nanfeng mandarin (Citrus reticulata cv. Blanco) in China (Peng et al., 2018). Also, $H$. uvarum was detected in the leaf galls formed by Pontania sawflies in four different plant species, especially at final stage of gall formation during July and September with an average relative abundance ranging from 1.21 to $9.45 \%$ (Glushakova and Kachalkin, 2017). Moreover, $H$. uvarum was detected as endophyte in leaves, flowers, immature and mature fruits of strawberry (Hall and Wilcox, 2018).

In general, grapevine can take up yeasts from the soil via roots and transport them through the vine to the stem and surface of grape berries in the pre-harvest season (Mandl et al., 2015). In this study, two genus only, Cladosporium and Alternaria were detected in all locations. Previous investigation found that the majority of fungal endophytic strains of grapevine included Cladosporium spp. and Alternaria spp. (Wijekoon and Quill, 2021). These genera are dominant and can be represented in all areas as endophytes in leaves and fruits of grape and they have been reported as a pre- and postharvest pathogen of concern in table grapes (Brum et al., 2012), they have also been detected as endophyte in different tissues of strawberry (Hall and Wilcox, 


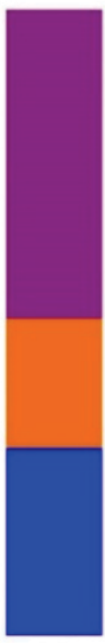

L1

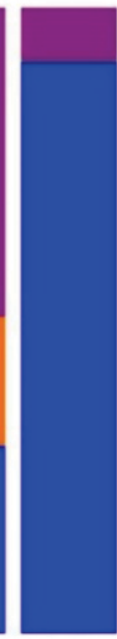

L2

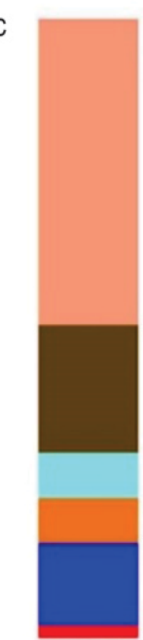

L1

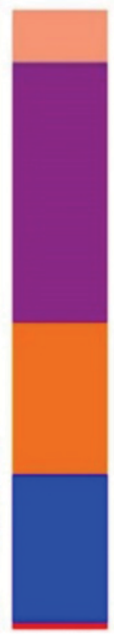

L2

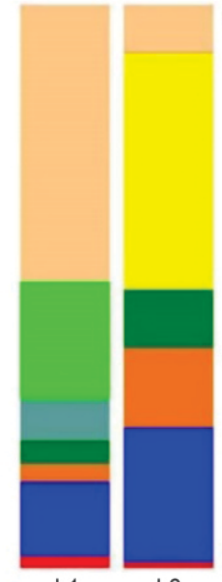

L1

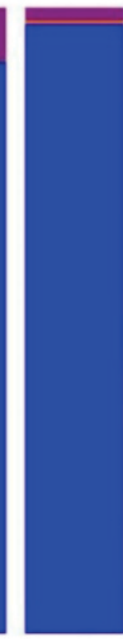

L3

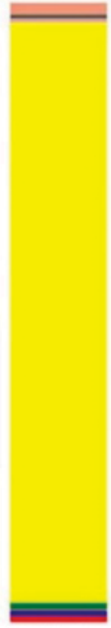

L3

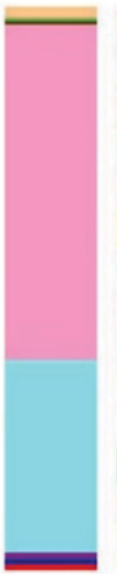

L3
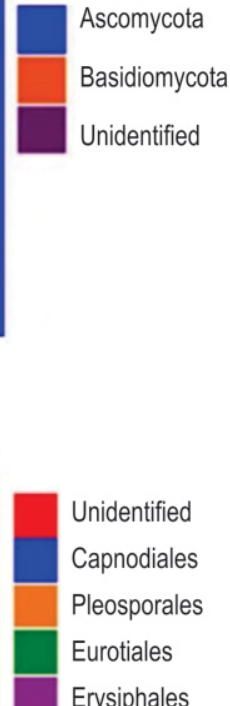

Saccharomycetales Hypocreales Sordariales Microstromatales Filobasidiales Unidentified

Unidentified

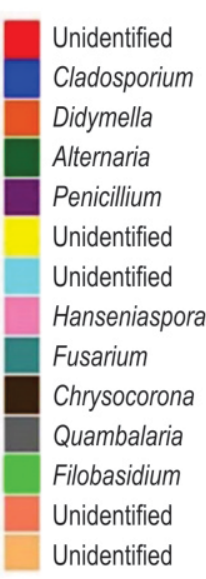

Unidentified b

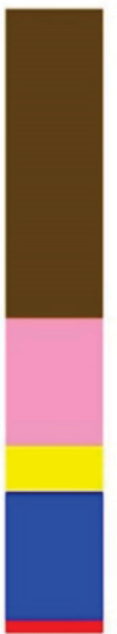

L1

d

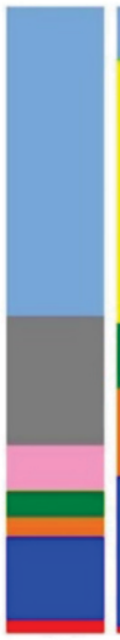

L1

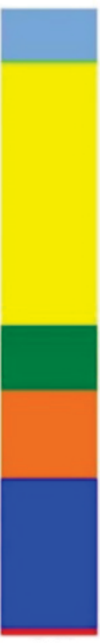

L2

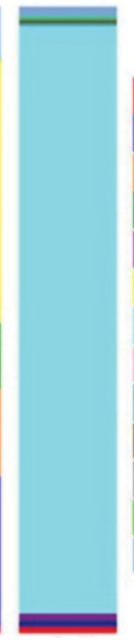

L3

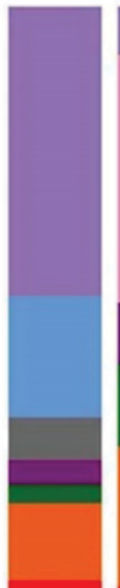

L1

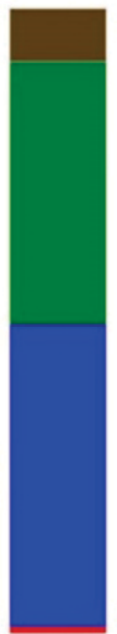

L2

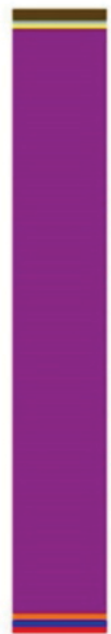

L3

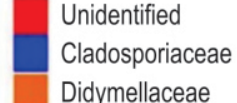

Didymellaceae

Pleosporaceae

Aspergillaceae

Erysiphaceae

Saccharomycodaceae

Nectriaceae

Chaetomiaceae

Quambalariaceae

Filobasidiaceae

Unidentified

Unidentified

Fig. 4: Relative sequence abundance of endophytic fungi detected in grape leaves collected from three different geographical locations $(L 1, L 2, L 3)$ ranging from phylum to species level. 
2018) and mangrove (Hamzah et al., 2018). Moreover, Cladosporium spp. may have a role in bioactive metabolite production due to its involvement in producing the anticancer compound L-asperaginase in Asperaceae family (Hatamzadeh et al., 2020). Fusarium genus in this study was detected at one location only although it is one of the commonly endophytic fungi genera in grapes and is known to produce a group of antibiotics called enniatins (Brum et al., 2012). Penicillium spp. was recorded as two species in this study at one location. It is a genus with interesting antagonistic activity against various pathogenic fungi due to the production of secondary metabolites with antibiotic activity (De Stefanoa et al., 1999), while the endophytic strains of Penicillium produce gibberellins to overcome salt stress (Leitao and Enguita, 2016). Basidomycota phylum represented Filobasidium magnum as the dominant species in the current study. Filobasidium spp. are ubiquitous in the vineyard environment and they are typically associated with grapes, phyllosphere and soil (Setati et al., 2012). In general, the presence and diversity of endophytic microorganisms, especially fungi can be affected by the phyllosphere of grapevine cultivars, plant treatments, agricultural practices, microclimatic conditions, soil properties, pathogen infections, plant physiology and anthropogenic factors (Varanda et al., 2016).

The results conclude the presence of diverse fungal communities in V. vinifera leaves at Taif region, Saudi Arabia. Metagenomic diversity showed differential richness of various taxonomic levels with pronounced dominance of specific phylum, genera, and species at different locations. Also, there was a location-specific taxonomic level. Some of the detected endophytic species will improve biocontrol management of Taify grapevine pests and enhance its organic production and quality either at preharvest or postharvest stages of production.

\section{Acknowledgment}

This study was funded by High Altitude Research Center, Taif University, Saudi Arabia. Grant No.: 1-440-6171.

\section{Add-on Information}

Authors' contribution: S.M. Sayed: Resources, Methodology, writing final draft; A.M. El-Shehawi: Data analysis, Formal analysis, Methodology, writing final draft; S.A. Elarnaouty: Formal analysis, writing final draft; S.A. Al-Otaibi: Resources, investigation, writing original draft; S.A. El-Shazly: Methodology, investigation, writing original draft; S.S. Alotaibi: Data analysis, Formal analysis; R. Ibrahim: Methodology, writing original draft; M.M. Ahmed: Data analysis, writing final draft; M. Elseehy: Methodology, writing original draft.

Research content: The research content is original and has not been published elsewhere

\section{Ethical approval: NotApplicable}

Conflict of interest: The authors declare that there is no conflict of interest.

\section{Data from other sources: NotApplicable}

Consent to publish: All authors agree to publish the paper in Journal of Environmental Biology.

\section{References}

Abdelfattah, A., M. Wisniewski, M.G. Li Destri Nicosia, S.O. Cacciola and L. Schena: Metagenomic analysis of fungal diversity on strawberry plants and the effect of management practices on the fungal community structure of aerial organs. PLOS ONE, 11, e0160470 (2016).

Brum, M.C.P., W.L. Araújo, C.S. Maki and J.L. Azevedo: Endophytic fungi from Vitis labrusca L. ('Niagara Rosada') and its potential for the biological control of Fusarium oxysporum. Gen. Mol. Res., 11, 4187-4197(2012).

Caporaso, J.G., J. Kuczynski and R. Knight: QIIME allows analysis of high-throughput community sequencing data. Nat. Meth., 7, 335$336(2010)$

Castañeda, L.E., T. Miura, R. Sánchez and O. Barbosa: Effects of agricultural management on phyllosphere fungal diversity in vineyards and the association with adjacent native forests. Peer J., 6, e $5715(2018)$.

De Stefanoa, S., R. Nicoletti, A. Milonea and S. Zambardinoa: 3-0Methylfunicone, fungitoxic metabolite produced by the fungus Penicillium pinophilum. Phytochemistry, 52, 1399-1401(1999).

Fahmi, A.I., M.A. Nagaty and A.M. El-Shehawi: Fruit quality of Taif grape (Vitis vinifera L.) cultivars. J. Amer. Sci., 8, 590-599 (2012).

Gao, F., J. Chen, J. Xiao, W. Cheng, X. Zheng, B. Wang and X. Shi: Microbial community composition on grape surface controlled by geographical factors of different wine regions in Xinjiang, China. Food Res. Int., 122, 348-360 (2019).

Glushakova, A.M. and A.V. Kachalkin: Endophytic yeasts in leaf galls. Microbiology, 86, 250-256 (2017).

Gonzalez, V. and M. Tello: The endophytic mycota associated with Vitis vinifera in Central Spain. Fungal Diver, 47, 29-42(2011).

Hall, M.E. and W.F. Wilcox: Identification and frequencies of endophytic microbes within healthy grape berries. Am. J. Enol. Vitic., ajev.2018.18033 (2018).

Hallmann, J. and R.A. Sikora: Toxicity of fungal endophyte secondary metabolites to plant parasitic nematodes and soil-borne plant pathogenic fungi. Eur. J. Plant Pathol., 102, 155-162 (1996).

Hamzah, T., S. Lee, A. Hidayat, R. Terhem, I. Faridah-Hanum and R. Mohamed: Diversity and characterization of endophytic fungi isolated from the tropical mangrove species, Rhizophora mucronata, and identification of potential antagonists against the soil-borne fungus, Fusarium solani. Front. Microbiol., 9, 1707 (2018).

Hatamzadeh, S., K. Rahnama, S. Nasrollahnejad, K.B. Fotouhifar, K. Hemmati, J.F. Whiteand and F.Taliei: Isolation and identification of L-asparaginase-producing endophytic fungi from the Asteraceae family plant species of Iran. Peer. J., 8, e8309. (2020).

Hernández-Hernández, L., J.T. Coutiño-Megchun, C.I. Rincón-Molina, V.M. Ruíz-Valdiviezo, J.M. Culebro-Ricaldi, R.I. Cruz-Rodríguez, H.H. Palomeque-Dominguez and R. Rincón-Rosales: Endophytic bacteria from root nodules of Ormosia macrocalyx with potential as plant growth promoters and antifungal activity. J. Environ. Biol., 39, 997-1005 (2018).

Kim, B.R., J. Shin, R. Guevarra, J.H. Lee, D.W. Kim, K.H. Seol, J.H. Lee, H.B. Kim and R. Isaacson: Deciphering diversity indices for a better understanding of microbial communities. J. Microbiol. Biotechnol., 27, 2089-2093 (2017).

Kioroglou, D., E. Kraeva-Deloire, L.M. Schmidtke, A. Mas and M.C. 
Portillo: Geographical origin has a greater impact on grape berry fungal community than grape variety and maturation state. Microorganisms, 7, 669 (2019).

Kuczynski, J., J. Stombaugh, W.A. Walters, A. González, J.G. Caporaso and R. Knight: Using QIIME to analyze 16S rRNA gene sequences from microbial communities. Curr. Protoc. Bioinfor., 36, 10.7.110.7.2(2011).

Leitao, A.L. and F.J., Enguita: Gibberellins in Penicillium strains: Challenges for endophyte-plant host interactions under salinity stress. Microbiol. Res., 183, 8-18 (2016).

Lindahl, B.D., R.H. Nilsson, L. Tedersoo, K. Abarenkov, T. Carlsen, R. Kjøller, U. Kõljalg, T. Pennanen, S. Rosendahl, J. Stenlid and H. Kauserud: Fungal community analysis by high-throughput sequencing of amplified makers - A user's guide. New Phytol., 199, 288-299 (2013).

Mandl, K., J. Schieck, K. Sikhavy-Richter, V. Schneider and H.P. Schmidt: Vines take up yeasts from soil and transport them through the vine to the stem and skins of grapes. Ithaka J., 349-355 (2015)

Mardis, E.R.: The impact of next-generation sequencing technology on genetics. Trends Genet., 24, 133-141(2008).

Martelli, G.P.: Directory of virus and virus-like diseases of the grapevine and their agents. J. Plant. Pathol., 96, 1-136 (2014).

Martin, V., M.J. Valera, K. Medina, E. Boido and F. Carrau: Oenological impact of the Hanseniaspora/Kloeckera yeast genus on wines-A Review. Fermentation, 4, 76 (2018).
Peng, X., Y. Wang, L.J. Tang, X.X. Li, Y.W. Xiao, Z.B. Zhang, R.M. Yan, H.L. Yang, J. Chang, B. Zhu and D. Zhu: Yeasts from Nanfeng mandarin plants: Occurrence, diversity and capability to produce indole-3-acetic acid. Biotechnol. Biotechnol. Equip., 32, 14961506 (2018).

Setati, M.E., D. Jacobson, U.C. Andong and F.F. Bauer: The vineyard yeast microbiome, a mixed model microbial map. PLOS ONE, 7, e52609 (2012).

Srivastava, S., P.S. Gupta, S. Lal and O.K. Sinha: Rapid identification of endophytic fungi of sugarcane (Saccharum spp. hybrid) using PCR-RFLP of rDNA. J. Environ. Biol., 38, 21-26 (2017).

Varanda, C.M.R., M. Oliveira, P. Materatski, M. Landum, M.I.E. Clara and M.R. Félix: Fungal endophytic communities associated to the phyllosphere of grapevine cultivars under different types of management. Fungal Biol., 120, 1525-1536 (2016).

White, T.J., T. Bruns, S. Lee and J. Taylor: Amplification and direct sequencing of fungal ribosomal RNA genes for phylogenetics. In: PCR Protocols: A Guide to Methods and Applications. (Eds.: M.A. Innes D.H. Gelfand, J.J. Sninsky and T.J. White). Academic Press, San Diego, CA, pp. 315-322 (1990).

Wijekoon, C. and Z. Quill: Fungal endophyte diversity in table grapes. Can. J. Microbiol., 67, 29-36 (2021).

Wu, S.C., C.S. Rau, H.T. Liu, P.J. Kuo, P.C. Chien, T.M. Hsieh, C.H. Tsai, J.F. Chuang, C.Y. Huang, H.Y. Hsieh and C.H. Hsieh: Metagenome analysis as a tool to study bacterial Infection associated with acute surgical abdomen. J. Clin. Med., 7, 346 (2018). 